\title{
Patterns and Determinants of Complementary and Alternative Medicine Practitioner Use among Adults with Diabetes in Queensland, Australia
}

\author{
Chi-Wai Lui, Jo Dower, Maria Donald, and Joseph R. Coll \\ School of Population Health, The University of Queensland, Herston Road, Brisbane, QLD 4006, Australia \\ Correspondence should be addressed to Chi-Wai Lui, c.lui@sph.uq.edu.au
}

Received 2 April 2012; Accepted 15 June 2012

Academic Editor: Jenny M. Wilkinson

Copyright () 2012 Chi-Wai Lui et al. This is an open access article distributed under the Creative Commons Attribution License, which permits unrestricted use, distribution, and reproduction in any medium, provided the original work is properly cited.

\begin{abstract}
There is evidence that complementary and alternative medicine (CAM) use is common among people with diabetes. The role of CAM in the treatment or management of diabetes is an emerging health issue given the potential side effects and benefits associated with the use of this kind of medicine. This paper examined patterns and determinants of CAM practitioner use in Queensland, Australia, using a large population-based sample of people with type 1 and type 2 diabetes. The study found that within a 12month period, $7.7 \%$ of people with diabetes used the services of CAM practitioners alongside or as a complement to conventional health care service. Younger age, female gender, a higher education, having private health insurance, and engagement in preventive health behaviours are significant predictors of individuals who are more likely to visit a CAM practitioner. There was no significant difference in CAM practitioner use between people with type 1, type 2 insulin requiring, or type 2 noninsulin requiring diabetes. The findings highlight the need for further research on the role of CAM in the prevention and management of diabetes.
\end{abstract}

\section{Introduction}

Diabetes mellitus is one of the key global health challenges. In 2008, 347 million people worldwide were estimated to have diabetes [1]. In Australia, an estimated total of 898,800 Australians had been diagnosed with diabetes in 2007-2008. Among these individuals, 87,100 had been diagnosed with type 1 diabetes and 787,500 with type 2 diabetes [2]. It has been projected that 3 million Australians will be living with diabetes by 2025 as a result of recent increases in the incidence of obesity and declines in mortality [3].

Diabetes is a chronic debilitating illness that requires regular monitoring and control. The disease often has an adverse impact on the patients' quality of life. The management of diabetes requires the use of extensive health care services and is both physically and emotionally demanding $[4,5]$. The degenerative nature of diabetes is such that the human and economic costs can increase dramatically with disease progression [6].

In recent years, there is a rising concern that many patients use complementary and alternative medicine
(CAM) to cope with the daily challenge of diabetes and the multiple complications that are often associated with this condition [7-9]. The use of CAM may have side effects and can interact with conventional diabetes treatment [7]. There is evidence that CAM use is common among diabetes patients across different age groups [10-16] and cultures [17-21]. A recent literature review found that the prevalence of CAM use among people living with diabetes ranges from $17 \%$ to $73 \%$ [22]. The wide variation in reported prevalence likely reflects differences in research or sample design, definitions of CAM (practitioner and/or self-prescribed medication), or measurements of CAM use (e.g., lifetime use or use over the previous 12 months) employed by different research projects [22]. Current research findings suggest that nutritional supplements, herbal medicines, nutritional advice, spiritual healing, and relaxation techniques are the most widely consumed CAM therapies among diabetic populations [22].

Despite the rise in interest in the use of CAM among people with diabetes, the knowledge base for this issue remains limited. This is especially the case in Australia, since 
to date most studies on this topic have been conducted in North America. A recent study of the use of CAM for the treatment of chronic illness using the National Health Survey database found that about $4 \%$ of Australian adults used CAM for their treatment of diabetes in 2004-2005 [23]. This paper contributes to our understanding of CAM use among Australians living with type 1 and type 2 diabetes by examining the prevalence, profile, and predictors of CAM practitioner use in the state of Queensland, Australia, using a large population-based sample of people with diabetes. The study focuses broadly on diabetic patients' use of CAM practitioners for any purpose whether related or not to their diabetes management.

\section{Materials and Methods}

2.1. Study Design and Sample. Data reported here are taken from the Living with Diabetes Study (LWDS), a five-year, prospective cohort study being conducted in the State of Queensland, Australia. The characteristics of the sample and methodology, including instrumentation, has been described in detail elsewhere [24], but briefly, the sample was recruited from the National Diabetes Services Scheme (NDSS), a government initiative that delivers diabetes-related products at subsidised prices to registrants and which covers up to $90 \%$ of the Australian population diagnosed with diabetes [2].

People were eligible to participate in the LWDS if they were aged 18 years or older, had been diagnosed with type 1 or type 2 diabetes (gestational diabetes was excluded), had a valid postal address recorded with the NDSS, and indicated on their NDSS registration that they were interested in receiving information about opportunities to participate in research. The sampling scheme intentionally oversampled in three areas of policy interest: an outer metropolitan area a new suburban development, and a coastal agricultural community. All eligible individuals from these three locations were invited to participate. In addition, a random sample of approximately one in six eligible individuals from the rest of the state was invited to participate.

A sample of 14,439 NDSS registrants, out of a possible 155,874 who satisfied the eligibility criteria, was invited to participate in the LWDS study. Of these eligible responders, completed questionnaires and signed informed consent forms were returned by 3,951 participants, yielding a participation rate at baseline of $29 \%$. The response rate for participants consenting to participate in the LWDS, while low, is consistent with research showing that participation rates in large cohort studies appear to be decreasing [25]. The response rate for the first follow-up (2009) survey, from which the results of this paper are drawn, was $88 \%(n=$ $3,360)$.

2.2. Measures. LWDS participants are mailed an annual self-report survey, which collects information on a range of health and well being issues. The survey covers eight areas including (i) demographic characteristics, (ii) clinical factors, including characteristics of diabetes, (iii) life-stylerelated factors, (iv) health-related quality of life, (v) self management, (vi) health care services utilisation, (vii) satisfaction with and quality of health care, and (viii) emotional well being.

2.2.1. CAM Practitioner Use. Participants were asked to specify from a list which health professionals they had seen in the last 12 months. Respondents were defined as CAM users if they indicated they had consulted an alternative or complementary health practitioner during that time. Respondents who did not consult a CAM practitioner were classified as non-CAM users.

2.2.2. Demographic Variables. In addition to age and sex, participants were asked about the highest educational qualification they had completed, their household income, marital status, employment status and whether they had private health insurance. Postcode of residence was used to classify area of residence as urban, inner regional, or other.

2.2.3. Disease Characteristics. Participants were classified according to the type of diabetes they had-type 1, type 2 insulin requiring, or type 2 noninsulin requiring. Selfreported age at diagnosis was used to determine duration of diabetes. Participants also reported their latest $\mathrm{HbAlc}$ result and the frequency of hypoglycaemic symptoms in the last month. Participants specified with which of a list of diabetesrelated complications they had been diagnosed. Participants were also asked to specify with which of a list of comorbid conditions they had been diagnosed.

2.2.4. Lifestyle Behaviours. Participants reported their alcohol use, smoking status, physical activity in the last week, and dietary factors, including milk use, salt use, type of spread used on bread, and intake of fruit, vegetables, fish, and red meat. Body mass index (BMI) was calculated using the standard equation of weight divided by height squared [26].

2.2.5. Health-Related Quality of Life. Participants completed the EQ-5D [27], which is a short questionnaire with five questions on mobility, self-care, pain, social activities, and anxiety/depression. The Audit of Diabetes Dependent Quality of Life (ADDQoL) [28] was also completed as a disease-specific measure of the impact of diabetes on the patient's quality of life across 19 domains.

2.2.6. Satisfaction with and Quality of Care. Participants rated their satisfaction with their diabetes treatment as well as their overall satisfaction with the health care system. Participants were also asked about the ease of access to their main health care provider and to specialists as well as how long on average they had to wait for an appointment with their regular general practitioner. Patients were classified according to whether or not they were frequent attendees of their main health care provider, with frequent attendance defined as 12 or more self-reported visits in the past 12 months. Finally, respondents completed the Patient Assessmentof Chronic Illness Care (PACIC) scale [29], which measures the extent to which patients report receiving care in line with best practice 
regarding patient activation, delivery system design/decision support, goal setting/tailoring, problem solving/contextual, and followup/coordination.

2.3. Statistical Analyses. The demographic, health status and diabetes characteristics of CAM practitioner users and nonusers were compared using chi-square tests for categorical variables and by logistic regression for continuous variables. All variables that were univariately associated with CAM practitioner use $(P<0.05)$ were considered as candidates for inclusion in a multiple logistic regression to predict CAM practitioner use. The multiple logistic regression was fit using a backward stepwise logistic model to determine the most parsimonious set of variables that independently predict CAM practitioner use. A stepwise multiple logistic regression yielded results identical to the backward selection model.

\section{Results}

Use of a CAM practitioner could be ascertained for 3,337 participants. The demographic and health status characteristics of respondents of the 2009 LWDS survey are described in Table 1. The median age was 64 (IQR 57-71) and the median duration of diabetes was 7 years (IQR 3-11). Over half $(54 \%)$ had diabetes-related complications, and $63.1 \%$ had a history of 2 or more either concordant or discordant comorbidities. Two hundred and fifty-eight (7.7\%) of the respondents reported at least one visit to a CAM practitioner in the previous 12 months. $5.5 \%$ made more than 1 visit to a CAM practitioner (see Table 2). Only 2 CAM users reported that they had not visited their GP in the past 12 months, suggesting that the vast majority of diabetic patients were using CAM as complementary to their health care rather than as an alternative to their traditional care.

The following demographic variables were univariately associated with CAM practitioner use: female gender $(P<$ $0.001)$, a younger age $(P<0.001)$, a higher level of educational attainment $(P<0.001)$, higher income $(P=0.010)$, and private health insurance $(P=0.009)$. Employment was also univariately associated with CAM use $(P<0.001)$ with retirees and those unable to work less likely and the unemployed more likely to visit a CAM practitioner.

No significant differences in CAM practitioner use between people with type 1 , type 2 insulin requiring, or type 2 noninsulin requiring diabetes were found. Four disease characteristics were univariately associated with CAM practitioner use including the presence of neuropathy $(P=0.015)$ and history of asthma $(P=0.003)$, anxiety $(P=0.004)$, or depression $(P=0.002)$ as a comorbidity. Univariately significant health and lifestyle factors included a higher number of serves of vegetables per day $(P<0.001)$ and fish $(P=0.028)$ eaten per week. Conversely, the use of butter rather than a butter substitute or no butter was associated with CAM practitioner use $(P=0.020)$. Being sufficiently active was associated with CAM practitioner use $(P=0.039)$ relative to those that are sedentary or insufficiently active. Reporting pain on the subscale of the EQ-5D measure of
TABLE 1: Demographic and health status characteristics of respondents of the 2009 LWDS survey.

\begin{tabular}{|c|c|c|}
\hline Variables & $N$ & $\begin{array}{c}n(\%) \text { or } \\
\text { median (IQR) }\end{array}$ \\
\hline Prevalence of CAM practitioner use & 3337 & $258(7.7)$ \\
\hline Sex & 3337 & \\
\hline Male & & $1828(54.7)$ \\
\hline Female & & $1509(45.2)$ \\
\hline Age & 3337 & \\
\hline $18-44$ year & & $225(6.7)$ \\
\hline $45-59$ year & & $909(27.2)$ \\
\hline $60-74$ years & & $1704(51.1)$ \\
\hline $75+$ years & & $499(15.0)$ \\
\hline Education level & 3291 & \\
\hline University degree & & $449(13.6)$ \\
\hline Certificate/diploma/trade & & $1005(30.5)$ \\
\hline Senior high school & & $472(14.3)$ \\
\hline Year 10 and below & & $1365(41.5)$ \\
\hline Employment status & 3277 & \\
\hline Employed (full/part time/casual/self) & & $1171(35.7)$ \\
\hline Home duties/carer & & $197(6.0)$ \\
\hline Unemployed (able to work) & & $66(2.0)$ \\
\hline Permanently ill & & $246(7.5)$ \\
\hline Retired & & $1597(48.7)$ \\
\hline Diabetes type & 3337 & \\
\hline Type 1 & & $144(4.3)$ \\
\hline Type 2: insulin requiring & & $615(18.4)$ \\
\hline Type 2: non insulin requiring & & $2578(77.3)$ \\
\hline Duration of diabetes (years) & 3265 & $7(3-11)$ \\
\hline High comorbidity $(2+)$ & 3330 & $2101(63.1)$ \\
\hline Number of complications & 3337 & $1(0-2)$ \\
\hline Obesity $(\mathrm{BMI}>30)$ & 3228 & $1604(49.7)$ \\
\hline Current smoker & 3303 & $320(9.7)$ \\
\hline
\end{tabular}

health-related quality of life was univariately associated with CAM practitioner use $(P=0.034)$ as was reporting a poorer diabetes-specific quality of life $(P=0.004)$ on the ADDQoL. Participants who reported that they were less satisfied with their diabetes treatment $(P=0.019)$ as well as those less satisfied with the health care system more generally $(P=$ 0.007 ) and those who reported that access to their main health care provider $(P=0.012)$ or access to a specialist $(P=0.047)$ was not easy were more likely to visit a CAM practitioner. In contrast, those who rated their quality of care more favourably on the PACIC were more likely to be CAM users $(P=0.018)$.

Results for the final logistic model are shown in Table 3. Twenty-one variables were univariately associated with CAM practitioner use and were entered as candidates into the multiple logistic regression model. Eight variables were retained as independent predictors of CAM practitioner use and included four socioeconomic characteristics, two health and lifestyle variables, and four health-related quality of 
TABLE 2: Distribution of CAM practitioner use by visits to the general practitioner, $n(\%)$.

\begin{tabular}{|c|c|c|c|c|c|}
\hline \multirow{2}{*}{ Frequency of CAM practitioner use } & \multirow[b]{2}{*}{$n(\%)$} & \multicolumn{4}{|c|}{ Visits to the GP } \\
\hline & & None & 1 to 4 & 5 to 11 & 12 or more \\
\hline None & $3079(92.3)$ & $60(1.9)$ & $1104(35.9)$ & $1258(40.9)$ & $657(21.3)$ \\
\hline 1 & $73(2.2)$ & $0(0.0)$ & $21(28.8)$ & $36(49.3)$ & $16(21.9)$ \\
\hline 2 to 6 & $125(3.8)$ & $2(1.6)$ & $44(35.2)$ & $50(40.0)$ & $29(23.2)$ \\
\hline 7 or more & $60(1.7)$ & $0(0.0)$ & $14(23.3)$ & $29(48.3)$ & $17(28.3)$ \\
\hline
\end{tabular}

life variables. In this diabetic patient group, younger age, more highly educated, and female gender are more likely to visit a CAM practitioner as those are with private health insurance. Diabetic patients who are CAM practitioner users can be classified as having a healthier lifestyle particularly in relation to their diet and exercise habits. Pain is an important determinant of CAM practitioner use. Those with a poorer health-related quality of life were more likely to use CAM services.

\section{Discussion}

This study examines the use of CAM practitioners using a large population-based sample of people with type 1 and type 2 diabetes. It offers a unique opportunity to investigate patterns and determinants of CAM use among this particular patient group in Australia. Our results indicate that about $8 \%$ of the LWDS respondents made at least one visit to a CAM practitioner in the previous 12 months. This level of CAM practitioner use is higher than the $4 \%$ use as reported by Armstrong et al. based on their analysis of the 2004-2005 National Health Survey database [23]. One possible explanation for this discrepancy is that the National Health Survey asked the respondents specifically about their use of CAM in the treatment or management of specific chronic conditions. In contrast, the LWDS survey did not differentiate between diabetes-specific CAM practitioner use and the use of CAM practitioners for nondiabetes conditions or general well being. As both the National Health Survey and LWDS did not record self-prescribed CAM use, the actual prevalence of CAM use among people with diabetes may be higher as studies on general population found that the use of self-prescribed CAM is common in Australia [30-32].

On the other hand, the level of CAM practitioner use we found in the LWDS sample is lower than the $17 \%-73 \%$ prevalence range as identified by a recent review on this topic [22]. This disparity can be explained by the fact that many previous studies had taken into consideration self-prescribed use of CAM products. The prevalence of CAM use amongst the LWDS sample should be higher if use of self-prescribed CAM products is also counted.

Our data reveal that younger age, female gender, and higher educational attainment are predictive of individuals more likely to visit CAM practitioners. This finding is broadly consistent with the profile of CAM users as identified in previous studies of general populations [33] as well as diabetes-specific populations [22]. The association of CAM practitioner use with higher income and private health insurance highlights the potential importance of the cost of CAM therapies in influencing consumption as CAM therapies or products are not currently covered by Medicare (Australia's universal health care system) or Australian Pharmaceutical Benefits Scheme (a federal government program providing subsidised prescription drugs to residents) so consumers pay the full expense when they choose these services/products.

As Table 2 demonstrates, the vast majority of this diabetes population visit CAM practitioners concurrently with their general practitioner. This reveals that people living with diabetes consider CAM therapies as a supplement rather than an alternative to mainstream treatment methods. Although the data highlights dissatisfaction with conventional health service as one of the predictors for uptake of CAM therapies, we found no evidence that CAM practitioner users receive suboptimal diabetes care and the use of CAM is not associated with $\mathrm{HbA1c}$ result or frequency of hypoglycaemic symptoms. However, as findings of previous studies indicated that the disclosure rate of CAM use to health care professionals remains very low $[34,35]$, the use of CAM alongside of conventional diabetes treatment may result in adverse reactions or drug interactions [36, 37]. This is an issue that requires further research and education.

The present study finds that people with poorer diabetesspecific quality of life are more likely to visit a CAM practitioner. In particular, the data suggest that pain is an important determinant of CAM practitioner use among people living with diabetes. This result suggests that people are willing to try CAM therapies when conventional treatment does not help. Studies of the general population have shown that chronic pain is one of the most commonly reported conditions for which people seek out and use CAM therapies $[38,39]$. The result of our study highlights the need to further investigate the role of CAM in controlling diabetes symptoms and enhancing patient's quality of life.

The results of our analysis show that CAM practitioner use is correlated with preventive and self-care behaviours, which may be part of a broader lifestyle that emphasizes on building health and resources for living. This finding is important as it highlights the potential role of CAM in health promotion and in facilitating a "structured" or lifestyle medicine approach to deal with chronic disease $[40,41]$. While there is evidence that for people living with diabetes CAM is used more for improving general well being than for treating diabetes-specific conditions [10, 11, 42], researchers should not overlook the potential and benefits of CAM in prevention and management of chronic disease and in achieving integrative care for diabetes. 





Several limitations of this study have to be acknowledged. First, analysis of aggregated NDSS data comparing participants of LWDS with nonparticipants indicated that individuals were more likely to participate if they were aged 50-69 years, and Indigenous Australians were less likely to participate; therefore, generalizing findings from this study to these populations must be undertaken with caution. Second, the survey only asked respondents about their visit of CAM practitioners and no information about use of selfprescribed CAM was recorded. As a result, the findings may underestimate the prevalence of CAM use among people with diabetes as there is evidence that self-prescribed CAM use is popular in Australia [30-32]. Finally, the interpretation of our findings is also limited by the fact that the health and CAM practitioner utilisation data is self-reported by the participants and is open to the effects of recall bias. However, such study limitations are outstripped by the insight gained through collecting and analysing such a large, populationbased sample of people living with diabetes.

\section{Conclusion}

This paper reports the findings of a study on the prevalence, profile, and predictors of CAM practitioner use amongst Australians living with type 1 and type 2 diabetes. The study highlights that $7.7 \%$ of people with diabetes in Queensland used services of CAM practitioner alongside or as a complement to conventional health care service and preventive care. The prevalence of CAM use amongst this group of individuals would be even higher if we take into consideration self-prescribed use of CAM products.

With a surge in the prevalence of diabetes in contemporary societies and a concurrent rise in consumer interest in CAM, there is an urgent need for research to examine CAM use behaviours and the wider role of CAM in the treatment and management of diabetes given the potential risks and benefits associated with the consumption of this kind of medicine. In particular, there is a need for in-depth qualitative research on the conception and experiences of CAM use in daily management of diabetes. The frequent use of a range of practitioner-based CAM amongst people with diabetes also highlights the importance of health care providers being cognisant of CAM ingredients and encouraging an open but critical dialogue on CAM use with their patients.

\section{Glossary}

ADDQoL: Audit of Diabetes Dependent Quality of Life

CAM: Complementary and alternative medicine

LWDS: $\quad$ Living with Diabetes Study

NDSS: National Diabetes Services Scheme

PACIC: Patient Assessment of Chronic Illness Care.

\section{Acknowledgment}

The Living with Diabetes Study is funded by Queensland Health.

\section{References}

[1] G. Danaei, M. M. Finucane, Y. Lu et al., "National, regional, and global trends in fasting plasma glucose and diabetes prevalence since 1980: systematic analysis of health examination surveys and epidemiological studies with 370 country-years and 2.7 million participants," The Lancet, vol. 378, no. 9785, pp. 31-40, 2011.

[2] Australian Institute of Health and Welfare, "Diabetes prevalence in Australia: an assessment of national data sources," Australian Institute of Health and Welfare, Canberra, Australia, 2009.

[3] D. J. Magliano, A. Peeters, T. Vos et al., "Projecting the burden of diabetes in Australia-what is the size of the matter?" Australian and New Zealand Journal of Public Health, vol. 33, no. 6, pp. 540-543, 2009.

[4] J. D. Piette and E. A. Kerr, "The impact of comorbid chronic conditions on diabetes care," Diabetes Care, vol. 29, no. 3, pp. 725-731, 2006.

[5] O. Solli, K. Stavem, and I. S. Kristiansen, "Health-related quality of life in diabetes: the associations of complications with EQ-5D scores," Health and Quality of Life Outcomes, vol. 8 , article 18, 2010.

[6] M. Brandle, H. Zhou, B. R. K. Smith et al., "The direct medical cost of type 2 diabetes," Diabetes Care, vol. 26, no. 8, pp. 23002304, 2003.

[7] J. Hart, "Diabetes and complementary therapies: research review and clinical applications," Alternative and Complementary Therapies, vol. 12, no. 6, pp. 263-267, 2006.

[8] C. Payne, "Complementary and integrative medicine: emerging therapies for diabetes, part 1," Diabetes Spectrum, vol. 14, no. 3, pp. 129-131, 2001.

[9] B. S. O'Connell, “Complementary and integrative medicine: emerging therapies for diabetes, part 2," Diabetes Spectrum, vol. 14, no. 4, pp. 196-197, 2001.

[10] T. A. Arcury, R. A. Bell, B. M. Snively et al., "Complementary and alternative medicine use as health self-management: rural older adults with diabetes," Journals of Gerontology —Series B, vol. 61, no. 2, pp. S62-S70, 2006.

[11] R. A. Bell, J. M. Stafford, T. A. Arcury et al., "Complementary and alternative medicine use and diabetes self-management among rural older adults," Complementary Health Practice Review, vol. 11, no. 2, pp. 95-106, 2006.

[12] N. E. Schoenberg, E. P. Stoller, C. S. Kart, A. Perzynski, and E. E. Chapleski, "Complementary and alternative medicine use among a multiethnic sample of older adults with diabetes," Journal of Alternative and Complementary Medicine, vol. 10, no. 6, pp. 1061-1066, 2004.

[13] B. Stanjkler, J. Radanovic, G. Colig et al., "Use of complementary and alternative medicine in children with type 1 diabetes," European Diabetes Nursing, vol. 5, no. 3, article 98, 2008.

[14] D. Arýkan, S. K. Sívríkaya, and N. Olgun, "Complementary alternative medicine use in children with type 1 diabetes mellitus in Erzurum, Turkey," Journal of Clinical Nursing, vol. 18, no. 15, pp. 2136-2144, 2009.

[15] K. Dannemann, W. Hecker, H. Haberland et al., "Use of complementary and alternative medicine in children with type 1 diabetes mellitus - prevalence, patterns of use, and costs," Pediatric Diabetes, vol. 9, no. 3, pp. 228-235, 2008.

[16] R. L. McCarty, W. J. Weber, B. Loots et al., "Complementary and alternative medicine use and quality of life in pediatric diabetes," Journal of Alternative and Complementary Medicine, vol. 16, no. 2, pp. 165-173, 2010. 
[17] N. Argáez-López, N. H. Wacher, J. Kumate-Rodríguez et al., "The use of complementary and alternative medicine therapies in type 2 diabetic patients in Mexico," Diabetes Care, vol. 26, no. 8, pp. 2470-2471, 2003.

[18] S. Ceylan, Ö. Azal, A. Taşlipinar, T. Türker, C. H. Açikel, and M. Gulec, "Complementary and alternative medicine use among Turkish diabetes patients," Complementary Therapies in Medicine, vol. 17, no. 2, pp. 78-83, 2009.

[19] H. Y. A. Chang, M. Wallis, and E. Tiralongo, "Use of complementary and alternative medicine among people with type 2 diabetes in Taiwan: a cross-sectional survey," EvidenceBased Complementary and Alternative Medicine, vol. 2011, Article ID 983792, 8 pages, 2011.

[20] S. S. Hasan, W. C. Loon, K. Ahmadi, S. I. Ahmed, and N. I. Bukhari, "Reasons, perceived efficacy and factors associated with complementary and alternative medicine use among Malaysian patients with diabetes mellitus," British Journal of Diabetes and Vascular Disease, vol. 11, no. 2, pp. 92-98, 2011.

[21] A. J. Khalaf and D. L. Whitford, "The use of complementary and alternative medicine by patients with diabetes mellitus in Bahrain: a cross-sectional study," BMC Complementary and Alternative Medicine, vol. 10, article 35, 2010.

[22] H. Y. Chang, M. Wallis, and E. Tiralongo, "Use of complementary and alternative medicine among people living with diabetes: literature review," Journal of Advanced Nursing, vol. 58, no. 4, pp. 307-319, 2007.

[23] A. R. Armstrong, S. P. Thiébaut, L. J. Brown, and B. Nepal, "Australian adults use complementary and alternative medicine in the treatment of chronic illness-a national study," Australian and New Zealand Journal of Public Health, vol. 35, no. 4, pp. 384-390, 2011.

[24] M. Donald, J. Dower, R. Ware et al., "Living with diabetes: rationale, study design and baseline characteristics for an Australian prospective cohort study," BMC Public Health, vol. 12, article 8, 2012.

[25] E. A. Nohr, M. Frydenberg, T. B. Henriksen, and J. Olsen, "Does low participation in cohort studies induce bias?" Epidemiology, vol. 17, no. 4, pp. 413-418, 2006.

[26] World Health Organization, Obesity: Preventing and Managing the Global Epidemic, World Health Organization, Geneva, Switzerland, 2000.

[27] R. Rabin and F. de Charro, "EQ-5D: a measure of health status from the EuroQol Group," Annals of Medicine, vol. 33, no. 5, pp. 337-343, 2001.

[28] C. Bradley, C. Todd, T. Gorton, E. Symonds, A. Martin, and R. Plowright, "The development of an individualized questionnaire measure of perceived impact of diabetes on quality of life: the ADDQoL," Quality of Life Research, vol. 8, no. 1-2, pp. 79-91, 1999.

[29] R. E. Glasgow, E. H. Wagner, J. Schaefer, L. D. Mahoney, R. J. Reid, and S. M. Greene, "Development and validation of the patient assessment of chronic illness care (PACIC)," Medical Care, vol. 43, no. 5, pp. 436-444, 2005.

[30] J. Adams et al., "A comparison of complementary and alternative medicine users and use across geographical areas: a national survey of 1, 427 women," BMC Complementary and Alternative Medicine, vol. 11, article 85, 2011.

[31] J. Wardle, C. W. Lui, and J. Adams, "Complementary and alternative medicine in rural communities: current research and future directions," The Journal of Rural Health, vol. 28, no. 1, pp. 101-112, 2012.

[32] P. Thomson, J. Jones, J. M. Evans, and S. L. Leslie, "Factors influencing the use of complementary and alternative medicine and whether patients inform their primary care physician," Complementary Therapies in Medicine, vol. 20, no. 1, pp. 45-53, 2012.

[33] G. Andrews, J. Adams, J. Segrott et al., "The prevalence and profile of CAM users and reasons for use," in Tradition, Complementary and Integrative Medicine: An International Reader, J. Adams, G. Andrews, J. Barnes et al., Eds., PalgraveMacmillan, London, UK, 2012.

[34] T. Dunning, "Complementary therapies and diabetes," Complementary Therapies in Nursing and Midwifery, vol. 9, no. 2, pp. 74-80, 2003.

[35] B. M. Shelley, A. L. Sussman, R. L. Williams, A. R. Segal, and B. F. Crabtree, “'They don't ask me so I don't tell them': patientclinician communication about traditional, complementary and alternative medicine," Annals of Family Medicine, vol. 7, no. 2, pp. 139-147, 2009.

[36] J. Lake, "Complementary, alternative, and integrative Rx: safety issues," Psychiatric Times, vol. 26, no. 7, pp. 1-4, 2009.

[37] L. A. Braun, E. Tiralongo, J. M. Wilkinson et al., "Adverse reactions to complementary medicines: the Australian pharmacy experience," International Journal of Pharmacy Practice, vol. 18, no. 4, pp. 242-244, 2010.

[38] P. M. Barnes, B. Bloom, and R. L. Nahin, Complementary and Alternative Medicine Use Among Adults and Children: United States, 2007, U.S. Department of Health and Human Services, Division of Health Interview Statistics, Centers for Disease Control and Prevention, National Center for Health Statistics, Hyattsville, MD, USA, 2008.

[39] D. W. Sibbritt and J. Adams, "Back pain amongst 8,910 young Australian women: a longitudinal analysis of the use of conventional providers, complementary and alternative medicine (CAM) practitioners and self-prescribed CAM," Clinical Rheumatology, vol. 29, no. 1, pp. 25-32, 2010.

[40] G. Egger and S. Egger, "Lifestyle medicine: the Australian experience," American Journal of Lifestyle Medicine, vol. 6, no. 1, pp. 26-30, 2012.

[41] G. J. Egger, A. F. Binns, and S. R. Rossner, "The emergence of "lifestyle medicine" as a structured approach for management of chronic disease," Medical Journal of Australia, vol. 190, no. 3, pp. 143-145, 2009.

[42] B. K. Lind, W. E. Lafferty, D. E. Grembowski, and P. K. Diehr, "Complementary and alternative provider use by insured patients with diabetes in Washington State," Journal of Alternative and Complementary Medicine, vol. 12, no. 1, pp. 71-77, 2006. 




The Scientific World Journal
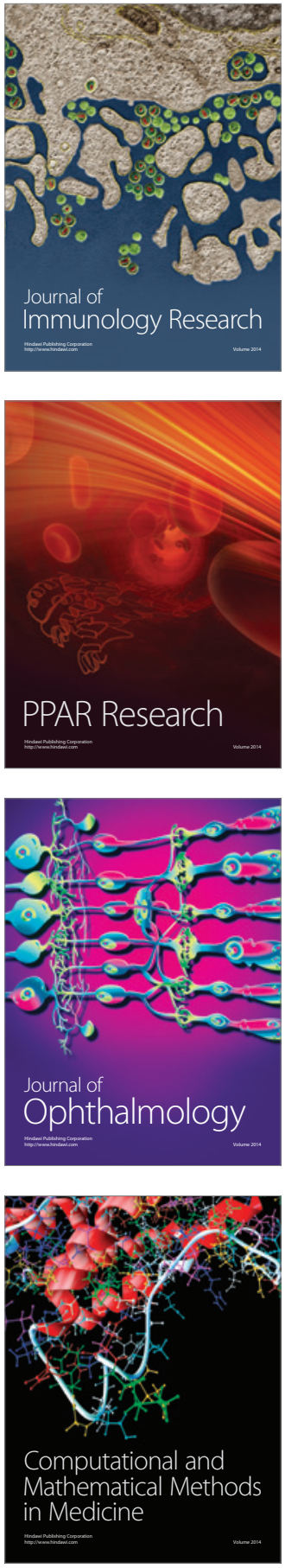

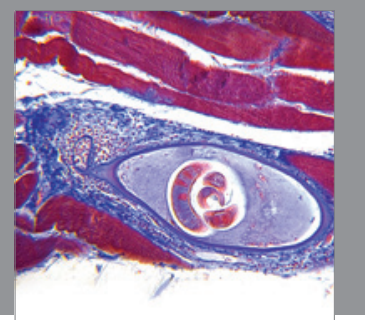

Gastroenterology

Research and Practice
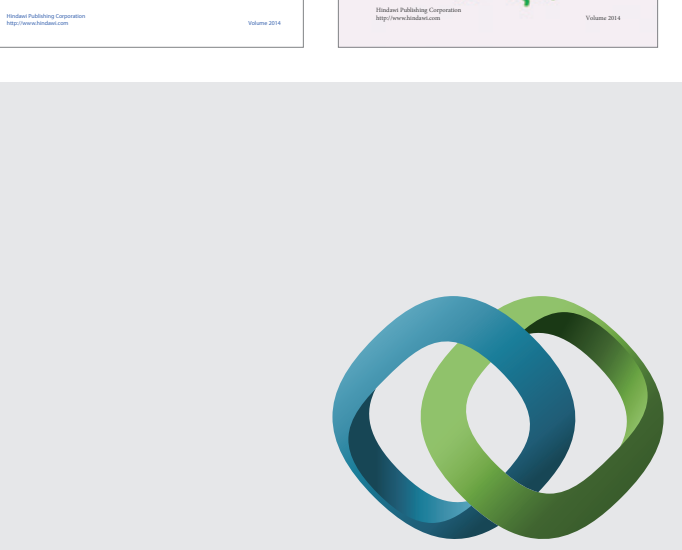

\section{Hindawi}

Submit your manuscripts at

http://www.hindawi.com
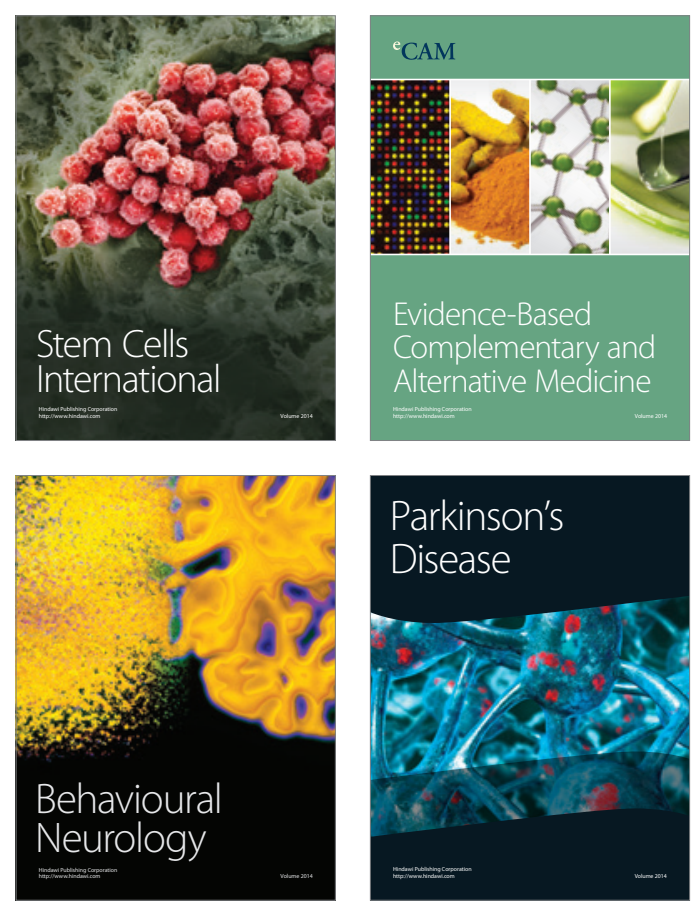

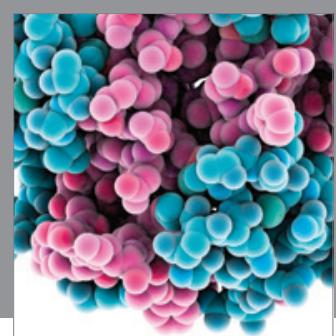

Journal of
Diabetes Research

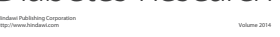

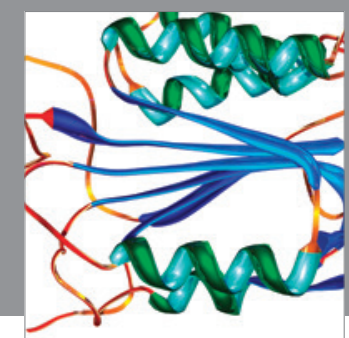

Disease Markers
